\title{
Le récepteur du mannose 6-P/IGF-II: un nouveau gène suppresseur de tumeur
}

Le récepteur mannose 6-phosphate/insulin-like growth factor (man 6-P/IGF-II) participe de façon classique au ciblage des hydrolases acides vers les lysosomes en se fixant spécifiquement aux résidus mannose 6phosphate présents sur la partie oligosaccharidique des enzymes lysosomales. Mais la découverte que le récepteur mannose 6-phosphate indépendant du calcium et le récepteur de l'IGF-II (insulin-like growth factor II) étaient la même protéine ont immédiatement suggéré pour ce récepteur un autre rôle que celui du ciblage cellulaire, à savoir un rôle de transmission du signal transmembranaire [1]. En fait, il a été montré que ce récepteur peut activer le facteur inhibiteur de croissance TGF- $\beta$ et agit sur la dégradation d'IGF-II, un facteur de croissance potentiel impliqué dans la tumorigenèse [2] et surexprimé dans les nodules métastatiques et les hépatocarcinomes humains. Ce rôle d'inhibiteur de croissance du récepteur d'IGF-II est dû également au fait qu'il distrait le facteur IGF-II de sa liaison au récepteur de l'IGF-I, le seul à envoyer à la cellule un signal de prolifération [3]. Les souris mutantes déficientes en récepteur d'IGF-II sont d'ailleurs caractérisées par une taille de $30 \%$ supérieure à la normale $\left(\mathrm{m} / \mathrm{s} n^{\circ} 10\right.$, vol. 11, p. 1483) $[4,5]$. La protéine liant IGF-II, IGFBP3, a une action similaire sur l'inhibition de la prolifération cellulaire en séquestrant les IGF. En outre, il vient d'être démontré que l'effet antiprolifératif de l'anti-œstrogène ICI 182780 dans les cancers du sein était associé à la synthèse d'IGFBP3 et d'IGFBP5 qui s'opposent à la liaison des IGF à leurs récepteurs $[6,7]$.
Étant donné la dualité de fonction du récepteur man 6-P/IGF-II, il était important de discriminer entre les deux fonctions de ce récepteur et d'attribuer des fonctions biologiques précises à l'une ou l'autre des propriétés de fixation de ce récepteur. L'analyse structurale puis génétique de ce récepteur ont permis de progresser dans cette voie.

Le récepteur man 6-P/IGF-II de 275 $\mathrm{kDa}$ possède une région aminoterminale de 44 acides aminés (aa), une région extracytoplasmique de 2269 aa, une région transmembranaire de 23 aa et un domaine carboxy-terminal cytoplasmique de 163 aa [2]. La région extracytoplasmique est formée de 15 domaines répétés de 147 résidus présentant une grande analogie de structure avec la partie extracytoplasmique du récepteur man 6-P dépendant du calcium, de $46 \mathrm{kDa}$ (CD-MPR)[1]. Enfin, le domaine répété 13 contient 43 aa présentant $50 \%$ d'identité avec la fibronectine de type II.

Le récepteur man 6-P/IGF-II étant une protéine bifonctionnelle, les différents domaines de fixation du récepteur ont ensuite été identifiés. Le man 6-P semble se fixer sur les domaines 3 et 9 extracytoplasmiques, alors que la zone de fixation d'IGF-II au récepteur est localisée au niveau du domaine répété 11 [8] et, plus particulièrement, des acides aminés 1508 à 1566 [9]. Une mutation ponctuelle remplaçant la thréonine 1572 par une isoleucine dans le même domaine 11 abolit complètement la fixation d'IGF-II [10] et une séquence de 10 acides aminés entourant cette isoleucine (résidus 1562 à 1572) est très conservée chez les mammifères, mais diverge de $85 \%$ à $78 \%$ dans le récepteur du poulet qui ne fixe pas l'IGF-II.

Plus récemment, plusieurs travaux ont permis d'impliquer le récepteur man 6-P/IGF-II dans la cancérogenèse. Des études génétiques ont d'abord montré que le récepteur de l'IGF-II ne présentait pas d'empreinte génomique parentale dans le foie de l'homme adulte alors qu'il existe une empreinte maternelle chez la souris [10]. Cette expression monoallélique du gène IGF-IIR chez la souris semble due à un mécanisme de compétition entre les gènes $H 19$ et IGF-IIR, dont l'empreinte parentale est en miroir, pour l'activation par une séquence activatrice commune $\left(\mathrm{m} / \mathrm{s} n^{\circ} 10\right.$, vol. 11, p. 1483) [11]. Chez l'homme, un déficit d'expression du gène IGF-IIR a été ensuite observé dans des carcinomes hépatocellulaires, amenant à l'observation de pertes d'hétérozygotie dans $70 \%$ de ces tumeurs et suggérant qu'un déficit partiel en récepteur man 6-P/IGF-II pourrait être un événement précoce au cours de l'hépatocarcinogenèse [12]. De plus, cette perte d'hétérozygotie est associée dans $25 \%$ des cas à trois types de mutations de l'allèle restant: l'une de type G:C-A:T crée un signal d'épissage alternatif aboutissant à la formation d'un récepteur tronqué qui supprime le domaine transmembranaire et les signaux de ciblage lysosomal; les deux autres substitutions sont du type C:G-T:A aboutissant à la substitution d'une glycine par une valine ou un acide glutamique et affectent la structure secondaire (feuillets plissés) du récepteur. 
Ces mutations sont localisées dans le domaine 10 , proche du site de fixation de l'IGF-II et renforcent l'hypothèse selon laquelle ce récepteur est, en effet, un gène suppresseur de tumeur [13].

Des expériences identiques concernant l'implication du récepteur man 6-P/IGF-II, dans les tumeurs du sein viennent d'être réalisées. Ces études montrent que $65 \%$ des patientes présentent une hétérozygotie informative du locus IGF-IIR (polymorphisme d'une séquence dinucléotidique répétée et délétion d'un tétranucléotide dans la région 3' non traduite du récepteur). Parmi ces malades, une perte d'hétérozygotie est observée dans $26 \%$ des carcinomes in situ et $33 \%$ des carcinomes invasifs. En outre, dans $40 \%$ de ces tumeurs également, l'allèle restant est muté comme cela a été montré dans les hépatocarcinomes [14]. Le gène du récepteur du man 6-P/IGF-II pourrait donc être considéré comme un gène suppresseur de tumeur intervenant dans les cancers du sein aussi bien que dans ceux du foie.

Ainsi le rôle de ce récepteur en tant que régulateur négatif de la croissance cellulaire, potentiel produit d'anti-oncogène, est-il bien établi. En revanche, son intervention dans le routage des enzymes lysosomales reste incertain, car les souris déficientes en ce récepteur, de petite taille, ne semblent pas avoir d'anomalies lysosomales $\left(\mathrm{m} / \mathrm{s} n^{\circ} 10\right.$, vol. 11 , p. 1483) [4].

J.F.R.

1. Kornfeld S. Structure and function of the mannose 6-phosphate insuline like growth factor II receptors. Аnnu Rev Biochem 1992; 61 : 307-30.

2. Nalk CG, Hanahan D. A second signal supplied by insulin-like growth factor II in oncogene induced tumorigenesis. Nature 1994; 369: 414-7.

3. Harel L. Les propriétés multiples des protéines de liaison des IGF (insulin-like growth factors): inhibiteurs et activateurs de croissance. Med Sci 1996; 12: 359-63.

4. Joshi R, Jami J. Invalidation chez la souris de gènes susceptibles d'être impliqués dans les diabètes non-insulinodépendants. Med Sci 1996; 12: 620-3.

5. Gilgenkrantz S. Facteurs de croissance et macrosomies: le temps des surprises. Med Sci 1996 ; $12: 815-6$

6. Huynh H, Yang X, Pollak M. Estradiol and antiestrogens regulate a growth inhibitory insulin-like growth factor binding protein 3 autocrine loop in human breast cancer cells. J Biol Chem 1996; 271 : 1016-21.

7. Huynh H, Yang X, Pollak M. A role for insulinlike growth factor binding protein 5 in the anti- proliferative action of the antiestrogen ICI 182780. Cell Growth Differ 1996; 7: 1501-6.

8. Dahms NM, Wick D, Brzycki-Wessell MA. The bovine mannose 6 -phosphate insulin-like growth factor II receptor. Localization of the insulin-like growth factor II binding site to domains 5-11. J Biol Chem 1994; 269 : 3802-9.

9. Schmidt B, Kiecke-Siemsen C, Waheeed A, Braulke T, Von Figura K. Localization of the insulin-like growth factor binding site to amino-acids 1508-1566 in repeat 11 of the mannose 6-phosphate insulin-like growth factor II receptor. J Biol Chem 1995; 270 : 14975-82.

10. Garmoudi F, Devi G, Slentz DH, Schaffer BS, Mac Donald RG. Truncated forms of the insulinlike growth factor II (IGF-II)/mannose 6-phosphate receptor encompassing the IGF-II binding site: characterization of a point mutation that abolishes IGF-II binding. Mol Endocr 1996; 10: 642-51.

11. Kalscheuer VM, Mariman EC, Schepens MT, Rehder H, Ropers HH. The insulin-like growth factor type-2 receptor gene is imprinted in the mouse but not in humans. Nature Genet 1993; 5 : 74-8.

12. De Souza AT, Hankins GR, Washington MK, Orton TC, Jirtle RL. M6P/IGF2R is mutated in human hepatocellular carcinomas with loss of heterozygosity. Nature Genet 1995; 11 : 447-9.

13. De Souza AT, Hankins GR, Washington MK, Orton TC, Jirtle RL. Frequent loss of heterozygosity on $6 \mathrm{q}$ at the mannose-6-phosphate/insulinlike growth factor II receptor locus in human hepatocellular tumors. Oncogene 1995; 10 : 1725-9.

14. Hankins GR, De Souza AT, Bentley RC, Patel MR, Marks JR, Iglehardt JD, Jirtle RL. M6P/IGF2 receptor: a candidate breast tumor suppressor gene. Oncogene 1996; 12: 2003-9.

\section{Colloque de Génétique Moléculaire et de Cytogénétique Humaine Sousse (Hôtel Tej Marhaba) - Tunisie 9-10 mai 1997}

Thèmes

Génétiques du Développement • Diagnostic anténatal • Génétique et cancer • Maladies héréditaires monofactorielles • Maladies héréditaires multifactorielles • Empreinte génomique.

Présentations orales et affichées

Les conférences ainsi que les résumés des travaux seront publiés dans un numéro spécial des archives de l'Institut Pasteur de Tunis.

Frais d'inscription

30 Dinars à régler par chèque à l'ordre de l'Amicale des Enseignants de la Faculté de Médecine de Sousse. Banque du Sud, Bab Jedid, Sousse - CCB nº 114044 136/2

Ce tarif inclut l'accès à la Salle de conférence, les documents et actes du congrès, les pauses café et les déjeuners.

Renseignements

Pr K. Dellagi, Laboratoire d'Immunologie et d'Immunopathologie, Institut Pasteur de Tunis, place Pasteur, 1000 Tunis, Tunisie. Tél.: 002161283022. Fax: 002161791833.

Pr A. Saad, Laboratoire de Cytogénétique et de Biologie de la Reproduction, CHU Farhat Hached, 4000 Sousse, Tunisie. Tél. : 002163221411. Pr. M. Fellous, Immunogénétique Humaine, Institut Pasteur Paris, 25, rue du Docteur-Roux, 75724 Paris Cedex 15, France.

Tél.: (33) 1456885 37. Fax: (33) 0140603153. 